\title{
Large and Small Scale Materials Testing of HT-9 Irradiated in the STIP Irradiation Program
}

\author{
P. Hosemann • Y. Dai • E. Stergar • H. Leitner • \\ E. Olivas • A.T. Nelson • S.A. Maloy
}

Received: 5 March 2010 / Accepted: 3 October 2010 /Published online: 2 November 2010

(C) The Author(s) 2010. This article is published with open access at Springerlink.com

\begin{abstract}
The Fuel Cycle R\&D (FCRD) initiative is investigating methods of burning minor actinides in a transmutation fuel. To achieve this goal, the fast reactor core materials must withstand very high doses. Small scale materials testing methods in addition to large scale materials testing allows one to gain more insight by providing more data on the same sample while being able to probe areas of interest which are not accessible otherwise. Furthermore, the sample volumes were so small that the tests could be considered a non destructive test since the amount of material needed is so small that a macroscopic structure would not be affected. Tensile testing, micro hardness testing and micro compression testing on focused ion beam (FIB) microscope manufactured pillars were performed on remaining parts of tensile test specimens tested and irradiated in the Spallation Target Irradiation Program (STIP). It is shown that the increases of yield strength measured by tensile testing, micro compression testing and micro hardness testing all showed the same trend. In addition FIB based techniques also allowed
\end{abstract}

P. Hosemann $(\bowtie) \cdot$ E. Stergar

Department of Nuclear Engineering, University of California, Berkeley, CA 94720, USA

e-mail: peterh@berkeley.edu

Y. Dai

Paul Scherrer Institute,

Villigen, Switzerland

E. Stergar $\cdot$ H. Leitner

University of Leoben,

Franz-Josef-Strasse 18,

8700 Leoben, Austria

E. Olivas · A.T. Nelson · S.A. Maloy

Los Alamos National Laboratory,

Los Alamos, NM 87545, USA fabrication of LEAP samples of such a small size that their residual activity was below detectable levels thus allowing them to be handled and analyzed in a manner comparable to inactive specimens.

Keywords Micro compression testing - Atom probe tomography (ATP) - Local electrode atom probe (LEAP) . Irradiated materials · HT-9 · Ferritic Martensitic Steel . Reactor materials $\cdot$ Small scale materials testing

\section{Introduction}

High energy particle (e.g. neutron) radiation can significantly alter materials properties. Phenomena like swelling, radiation induced embrittlement, creation of new isotopes, etc. can occur in materials exposed to a neutron flux at various conditions (temperature, dose, environment, etc). The evolution of physical or mechanical properties of materials is due to changes in the microstructure such as void formation, increase in dislocation density, formation of new phases, etc. Irradiation of materials can cause the formation of new isotopes such as Co-60 and Mn-54 in structural steels. The presence of these radioactive isotopes in the sample material makes the handling and measurements on these materials difficult and expensive.

Depending on the above mentioned specific irradiation conditions, target atoms can be transformed into different isotopes. Therefore it is difficult to establish general rules of a total activity applicable to all structural materials. However, for similar alloys (e.g. F/M steels containing the elements $\mathrm{Fe}, \mathrm{Cr}, \mathrm{C}$ ) and similar radiation conditions (e.g. high energy/current proton beam up to 20dpa as described in 2.1) the total number of isotopes created is comparable. In recent years the volume of sample material needed in 
order to perform a full assessment of the materials properties has been reduced significantly through maturation of small scale mechanical testing techniques. Today nanometer to micron sized samples can be used to determine microstructure, mechanical properties and composition. Reducing the volume of radioactive material needed for investigations relaxes handling and transportation restrictions on alloys of interest which both reduces costs and improves safety.

In this work, a conventional high-chromium nuclear steel (HT-9) was irradiated in the Spallation Target Irradiation Program STIP [1]. HT-9 is considered for in core structural components in the USA because of its substantial dataset on radiation induced property changes [2-5]. In addition, the performance of HT-9 in contact with lead bismuth eutectic coolants has been examined in relevant fast reactors environments [6,7]. After the actual STIP irradiation the samples were stored for a period of 62 month in order to allow for a decay of the shorter lived isotopes. This allowed for handling of samples outside of a hot cell in a controlled and properly shielded area. The specific allowable radioactivity levels were site specific depending on local regulations. However, samples with an activity of $3.6 \mathrm{mSv}$ at $10 \mathrm{~cm}$ could be handled. Gamma spectroscopy and dose measurements were performed to assess the amount of relevant radioactive isotopes in the material and to estimate the total amount of isotopes in a small scale sample such as local electrode atom probe or micro pillars. After characterizing the samples activity, both micromechanical testing (nanoindentation, micro compression testing) and local electrode atom probe measurements were performed.

\section{Experimental}

Ferritic/Martensitic steel (HT-9) with the nominal composition Fe-11.8Cr-0.2C-0.2Si-0.5Mn-0.5W-0.3V-1.0Mo$0.5 \mathrm{Ni}(\mathrm{wt} \%)$ was irradiated in the STIP III irradiation program. The complete experiment, sample preparation, and irradiation conditions are discussed in detail elsewhere [8] but the most relevant details are summarized below.

\section{The STIP Irradiation Parameters}

STIP-III was carried out in SINQ Target-5 which was irradiated through 2002 and 2003. The irradiation ended in December 2003. A total proton charge of $10.8 \mathrm{Ah}$ was received, which is slightly more than that of STIP-II $[9,10]$. A typical STIP irradiation takes 18 months. The accumulated dose is calculated using LAHET and MCNP-X codes. Temperature was monitored using thermocouples on the sample rods and post irradiation autoradiography was used to confirm dose calculations. The maximum irradiation dose reached in STIP 3 was about $25 \mathrm{dpa}$, which is the highest dose obtained from the STIP programs so far. Figure 1(a and b) illustrate the setup of the actual STIP irradiation program. Selected target rods (typically filled with lead) were removed and replaced with sample rods. These sample rods contain a large variety of different sample shapes as required for various post irradiation

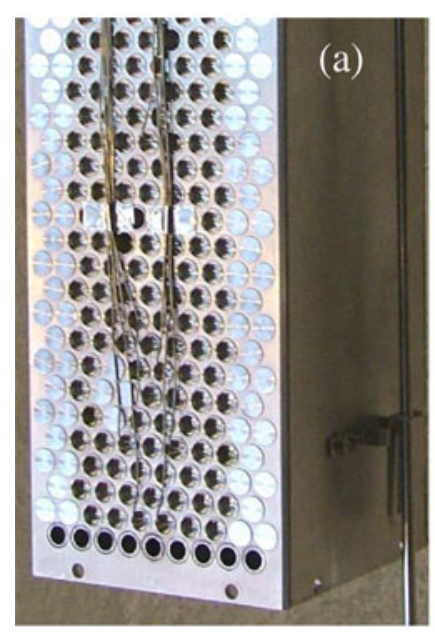

(b)

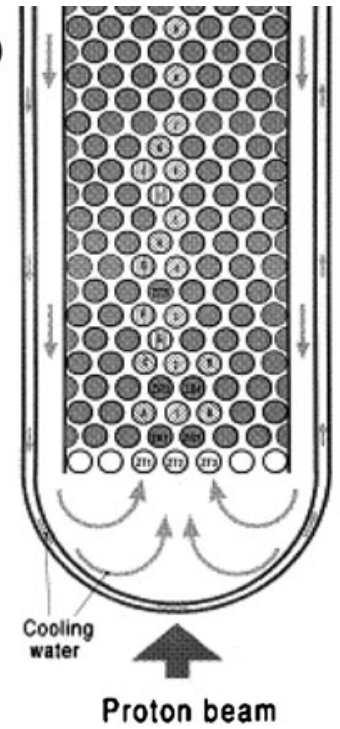

(c)

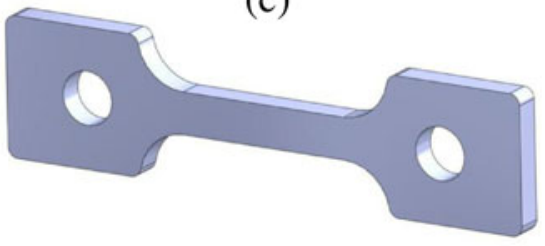

(d)

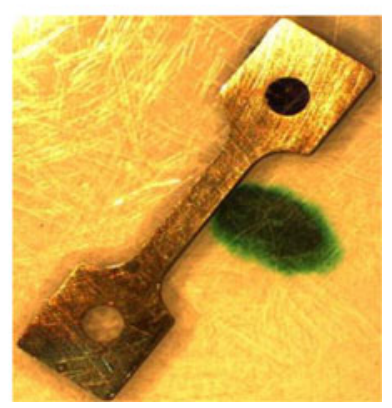

Fig. 1 STIP target (a) and (b) [3, 4], S1 sample design (c) and photograph of the sample in the hot cell (d) 
testing. Tensile samples were tested and analyzed in March 2009 following the 63 month cooling period. All dose measurements reported were done after this cooling time.

\section{Tensile Testing of the STIP Samples}

The tensile specimens exposed in STIP and tested after irradiation had a gauge section of $5 \mathrm{~mm} \times 1.2 \mathrm{~mm} \times$ $0.75 \mathrm{~mm}$, as shown in Fig. 1(c and d). The same sample geometry as described in $[9,10]$ was used in all STIP irradiation campaigns. Because of space restrictions the tensile samples are not ASTM standard samples [1]. However, miniaturizing of mechanical specimens is widely used in the nuclear field [11]. Before tensile testing the samples were cleaned and polished using a 600 grit silicon carbide grinding paper to ensure a clean and defect free surface since slight surface oxidation in the STIP environment can be found. Tensile tests were performed on a Zwick tensile testing machine. The tests were conducted at $300^{\circ} \mathrm{C}$ and $400^{\circ} \mathrm{C}$ at a nominal strain rate of $0.001 / \mathrm{sec}$. Mechanical testing was performed in a furnace which was heated from the outside and contained inert gas. To ensure homogeneous sample temperature the entire furnace interior was held at test temperature. The temperature was monitored using a thermocouple on the sample. Sample test temperatures were chosen to match the nominal irradiation temperature in an attempt to capture mechanical performance at operating conditions. Matching the test temperature to that of the irradiation allows for the assumption that defect annihilation would be largely limited to that which had occurred in the actual beam condition. Due to the fact that only one sample per irradiation condition was available, only a single temperature condition could be tested. This significant limitation clearly illustrates the need for development of different testing methods so a single sample can provide multiple data points to relay information on physical, mechanical, and structural property evolutions.

\section{Small Scale Sample Preparation}

The tabs (end pieces) remaining from tensile specimens after testing were used to perform the post irradiation micro scale mechanical testing and LEAP characterization. The samples were polished from both sides in order to remove surface damage after the tensile testing. Because the grips hold the sample in place and distribute the stress homogeneously over the entire grip surface, it was assumed that no plastic deformation of the samples took place and that the small scale tests still examined the same materials state as the macroscopic tests.
The samples were polished using 4000 grit silicon carbide paper followed by colloidal silica soft cloth polish using a Minimet polisher to reduce the risk of contamination. Subsequently these samples were placed in the FIB dedicated for active materials. Micro pillars were roughed using $24 \mathrm{nA}$ beam current followed by $80 \mathrm{pA}$ as a final step. In order to achieve a straight side of the pillars the sample was tilted $2^{\circ}$ towards the $\mathrm{Ga}$ beam for the last milling step. The pillars fabricated were $\sim 8 \times 8 \mu \mathrm{m}$ square and $16 \mu \mathrm{m}$ tall similar to pillars described previously $[12,13]$. Between three and four pillars per sample were produced. Each pillar was labeled in such a way that they could be distinguished from one and other using an optical microscope within the nanoindenter. This ensured unambiguous assignment of the exact dimensions of each pillar for the nanoindenter measurements.

After fabricating the micro pillars, LEAP needles were cut next to the pillars using the same FIB instrument. Initially a $1 \mu \mathrm{m}$ wide and $15 \mu \mathrm{m} \times 10 \mu \mathrm{m}$ foil was cut out of the sample surface followed by one out of two different preparation techniques:

- Initially foil production began with a $1 \mu \mathrm{m} \times 1 \mu \mathrm{m} \times$ $10 \mu \mathrm{m}$ slice cut from the foil and transported to the LEAP sample holder stubs [14-16] using the micro manipulator. The sample setup was shaped in a key and slot shape to ensure a good connection. Carbon was deposited on the connection using the gas injector systems (GIS) to ensure a good and stable joint. It was found that carbon gives a similar joint as Pt. While time consuming, this technique was determined to result in a stable connection and safe sample transport. The principle steps of this method are shown in Fig. 2(a-d).

- The second technique instead transported the entire foil to the sample holder stub once it was removed from the sample. Then the end of the foil was mounted on the stub by depositing carbon on the sample-stub joint after which the foil was cut so the LEAP needle was attached to the holder. This is a quicker way of mounting the sample but the joint between the actual sample and the Si holder might not be as stable as the initially described method above. The unique steps of Method 2 are illustrated in Fig. 2(e-f).

After mounting a $1 \times 1 \mu \mathrm{m}$ sample on the stubs, the sample holder was tilted towards the $\mathrm{Ga}$ beam so the feature milling tool on the Zeiss FIB could be used to finally shape the needle as shown in Fig. $2(\mathrm{~g})$.

\section{Small Scale Mechanical Testing}

The micro pillar testing was performed in an MTS nanoindenter. A lead shielded sample holder was designed for sample handling and to protect electronic components within the machine from radiation. The sample was loaded at a rate 

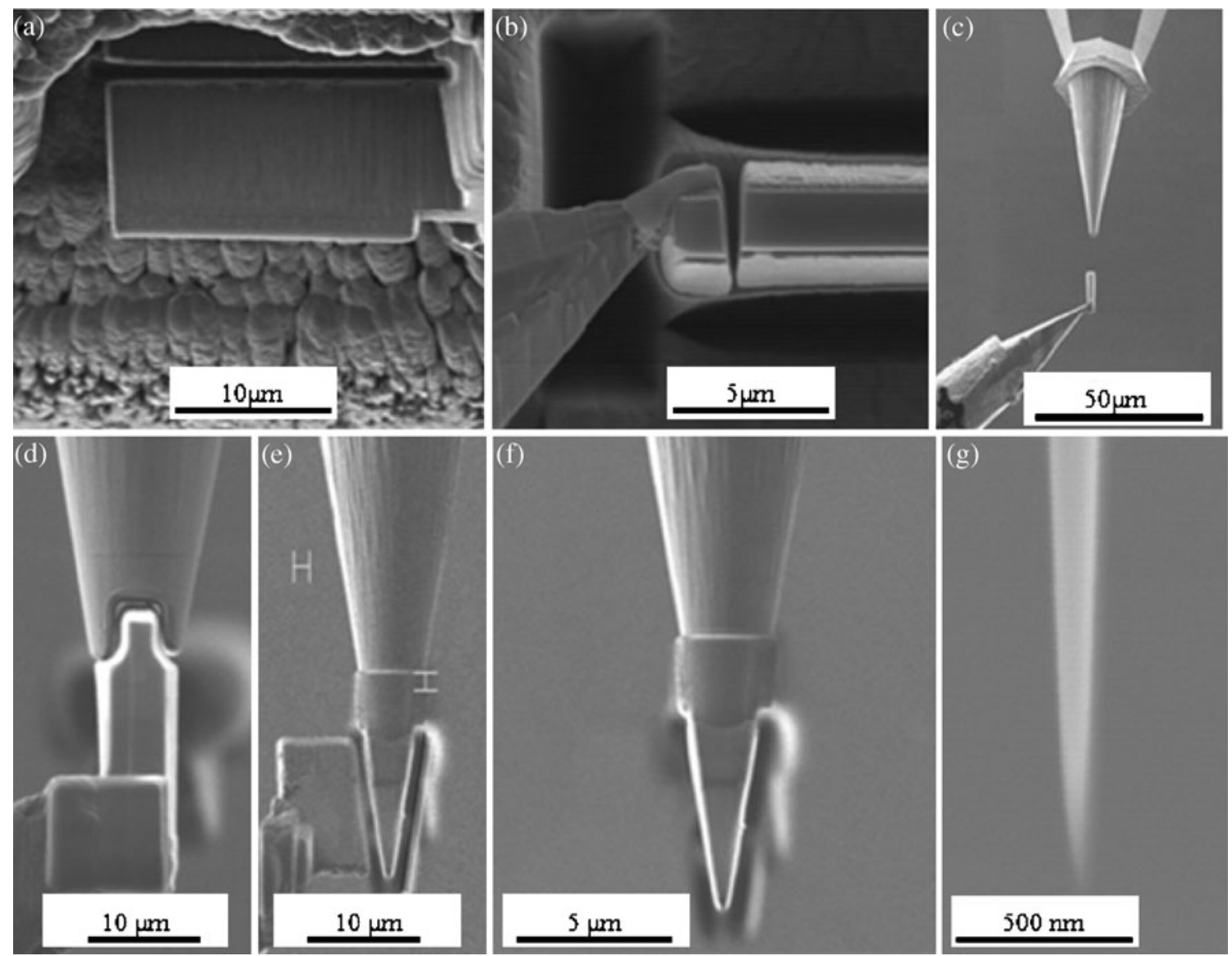

Fig. 2 Manufacturing of LEAP samples. Method 1: fabricating a $1 \mu \mathrm{m}$ wide foil (a), cutting a $1 \mu \mathrm{m} \times 1 \mu \mathrm{m}$ strip of the foil (b), transporting it to the LEAP sample holder (c) and manufacturing the key slot joint (d). Method 2: transporting the entire foil to the sample stub and welding the sample on the stub (e), The welded LEAP sample (f) and final sharpened needle (g)

of $0.2 \mathrm{mN} / \mathrm{sec}$. Each pillar was loaded to a different total displacement with a maximum of $3.5 \mu \mathrm{m}$. A comparison between the macroscopic tensile test and a micro compression test is shown in Fig. 3. The micro compression tests were performed as first suggested by Uchic et al. [17], except that the pillars were fabricated in a square shape similar to that employed by Kiener et al. [18]. Square pillars allow a simpler FIB procedure while being able to keep the sides of the pillar parallel without taper due to the fact that the sample tilt towards the ion beam can be adjusted accordingly.

The micro hardness testing was performed using a standard Vickers hardness indenter with an optical microscope. Ten indents with a test load of $50 \mathrm{~g}$ and $10 \mathrm{~s}$ dwell were performed on each sample.

\section{Local Electrode Atom Probe Measurements}

The final prepared LEAP samples were too small to have any measurable residual radioactivity and therefore could be transported easily. The samples were analyzed using an IMAGO LEAP 3000X-HR system in voltage mode. At
$46.4 \mathrm{~K}$ and $20 \%$ pulse fraction using $200 \mathrm{kHz}$ pulse repetition rate and 0.005 atoms per pulse. Several samples were characterized using this method. Reconstruction and post measurement analysis were performed using the software IVAS from IMAGO [19].

\section{Results and Discussion}

Dose Rate and Isotope Estimations on the STIP Samples

Gamma spectroscopy was performed on the samples from the STIP III irradiation in 2009, 63 months after the irradiation ended. The highest dose rate measured was 3.6 $\mathrm{mSv} / \mathrm{h}(360 \mathrm{mR} / \mathrm{h})$ at $10 \mathrm{~cm}$. This dose rate is on the high end of what can be handled outside of a hot cell at the Paul Scherrer Institute.

Table 1 shows the results of the gamma spectroscopy performed on the HT-9 samples. The total sample volume was $0.03049 \mathrm{~cm}^{3}$ per tensile sample. The total amount of radio isotopes in this volume is shown in Table 1. 


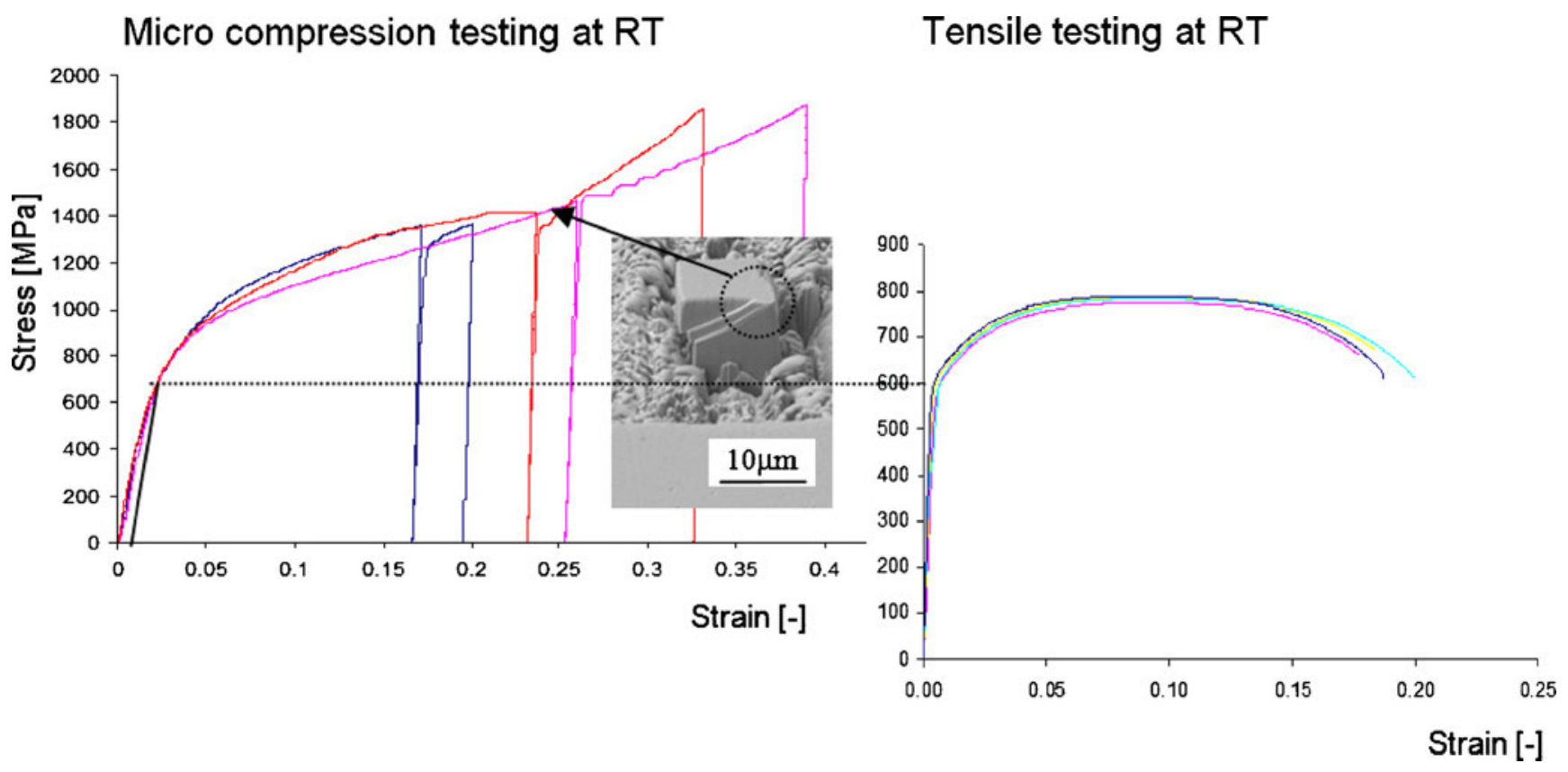

Fig. 3 Micro compression testing data and tensile testing data on a control sample of HT-9 performed at room temperature. Both methods show similar yield stresses

Based on these measurements the following estimations for LEAP samples were made:

The entire tensile sample has an activity of $7.6 \times 10^{7} \mathrm{~Bq}$, leading to volumetric activity rate of $2.49 \times 10^{9} \mathrm{~Bq} / \mathrm{cm}^{3}$. A rather large FIB machined LEAP sample is a cone with a $3 \mu \mathrm{m}$ height, $1 \mu \mathrm{m}$ base diameter and volume of $0.785 \times$ $10^{-12} \mathrm{~cm}^{3}$. Therefore, a conservative activity of $0.0019 \mathrm{~Bq}$ can be expected for a LEAP needle.

The concern in LEAP analysis of active materials is deposition of radioactive material within the equipment itself, resulting in contamination. A straightforward calcu- lation can be used to illustrate the negligible contamination concern presented by this technique. A background activity (as measured at Los Alamos National Laboratory) of $200 \mathrm{dpm}$ (disintegrations per minute) equates to $3.3 \mathrm{~Bq}$. 1700 LEAP samples combined would then be needed to produce a total activity which is equal to the background. In the unlikely case that the complete needle is ablated and two radioactive LEAP samples are examined each day, it would take $\sim 2.3$ years in order to reach the background level. Furthermore, the isotope present here with the longest half-life is Co-60 (5.27 years). By the time 1700 LEAP
Table 1 Gamma spectroscopy results on dose rate measurements on the STIP irradiated samples

The activity and total dose on a single LEAP sample is calculated as well as the number as LEAP needles needed in order to be above background. "M. to low" refers to a count rate too low to determine an accurate activity.

\begin{tabular}{lll}
\hline Isotope & Total activity measured [Bq] & Activity/LEAP needle [Bq] \\
\hline Na-22 & $6.5610^{5}$ & $1.6810^{-5}$ \\
Mn-54 & $5.0510^{7}$ & $1.2910^{-3}$ \\
Co-57 & $7.0410^{5}$ & $1.810^{-5}$ \\
Co-58 & M. to low & M. to low \\
Co-60 & $2.1910^{7}$ & $5.610^{-4}$ \\
Tc-95 & M. to low & M. to low \\
Hf-172 & $4.5710^{5}$ & $1.1710^{-5}$ \\
Lu-172 & $7.3610^{5}$ & $1.8810^{-5}$ \\
Lu-173 & $1.0610^{6}$ & $2.7910^{-5}$ \\
Re-186 & M. to low & M. to low \\
Total activity [Bq] & $7.610^{7}$ & $1.9510^{-3}$ \\
Total dpm \# LEAP needles until $200 \mathrm{dpm}$ & $4.5610^{9}$ & $1.7110^{-1}$ \\
& & $17110^{3}$ \\
mSv/h & 3.6 & $9.2810^{-11}$ \\
\hline
\end{tabular}


samples are measured a fraction of the deposited isotopes will have already decayed and more samples could be measured before the detectable limit is reached.

Co-60 is the isotope with the longest half life and the highest energy gamma emitter produced in significant quantities within these samples. Based on the measurements performed here it was found that roughly 145,000 Co-60 atoms $\left(2 \times 10^{-4}\right.$ atomic percent $)$ are present in one LEAP sample ( $3 \mu \mathrm{m}$ long $1 \mu \mathrm{m}$ diameter cone). For this estimation homogeneous distribution of Co-60 with an activity of $41,800 \mathrm{GBq} / \mathrm{g}$ in the steel is assumed. Considering that a total of 34 Million Co-60 atoms are needed to measure a half-life $(10 \mathrm{~h}$ count time, $3 \%$ detector efficiency, $1 \%$ accuracy and $200 \mathrm{dpm}$ background) the amount of Co60 present in one LEAP needle is not enough material to determine even a half-life.

In addition to analysis within the LEAP instrument itself, transportation and handling concerns are also greatly relaxed when dealing with the small volumes of prepared needles. The following dose estimations are made by disregarding the gamma spectroscopy measurement and only considering the dose rate as the limiting factor while using $0.06 \mu \mathrm{Sv} / \mathrm{h}$ as the detectable limit $(0.06 \mu \mathrm{Sv} / \mathrm{h}$ is the background at Los Alamos National Laboratory). It has been found that one LEAP sample has a total dose rate of $9.3 \times 10^{-8} \mu \mathrm{Sv} / \mathrm{h}$ at $10 \mathrm{~cm}$ distance. This leads to the conclusion that nearly 650,000 LEAP needles are needed in order to measure a dose rate above background in $10 \mathrm{~cm}$ distance.

The same estimations can be performed for micro mechanical tests. Micro pillars could be cut from the original sample and placed on an "anvil" and transported to a nanoindenter and tested. In this and previous work $[6,13]$ it has been shown that a sample of $8 \times 8 \times 16 \mu \mathrm{m}$ is sufficient to perform micro mechanical tests on irradiated $\mathrm{F} / \mathrm{M}$ materials. Using this volume as a comparison point, one micro pillar cut from a STIP tensile sample has $2.55 \mathrm{~Bq}$ which is again just below background levels. One micro pillar would have a dose rate of $1.2 \times 10^{-4} \mu \mathrm{Sv} / \mathrm{h}$ at $10 \mathrm{~cm}$, still well below the detectable dose rate limit for a Geiger counter.

The above sample calculations are useful to illustrate not only the particular margins for safety within this experiment and particular materials, but also can be extended to realize the utility of small sample test techniques when applied to evaluation of irradiated materials. One of the largest concerns to structural alloy qualification campaigns is the time and expense of post-irradiation examination. Even following removal from the irradiation facility, cask transport and hot cell examination of conventional specimen sizes demands an appreciable commitment. Production of small samples of the variety explored in this study at facilities such as PSI or LANL allows for a greater variety of analysis techniques to be performed either elsewhere on site, outside the added expense of substantial radiological controls, or even transported to other institutions. This will enhance both the depth and efficiency of analysis possible on irradiated materials as well as encourage collaborations in the field.

\section{Tensile Test Results on the STIP Samples}

Figure 3(a) presents results obtained from micro compression testing on non irradiated samples. Results from a control non irradiated control sample are shown in Fig. 3(b). Both tests were performed at room temperature. A comparison of the different tests exhibited a good agreement. Both yield stresses (YS) are found to fall the range of $600-650 \mathrm{MPa}$, as shown by the horizontal line in Fig. 3(a and b).

Several tensile test results on the HT-9 samples irradiated in STIP III as well as results from test samples are depicted in Fig. 4. The control sample tested at lower temperature $\left(300^{\circ} \mathrm{C}\right)$ shows higher YS but a lower uniform elongation than the control sample tested at higher temperature $\left(400^{\circ} \mathrm{C}\right)$.

The sample irradiated up to $15.2 \mathrm{dpa}$ and containing $1010 \mathrm{appm} \mathrm{He}$ with an average irradiation temperature of $451^{\circ} \mathrm{C}$ and a test temperature of $300^{\circ} \mathrm{C}$ shows the highest YS and a significant reduced elongation. The sample with the highest dose of $19 \mathrm{dpa}$, containing $1590 \mathrm{appm} \mathrm{He}$, irradiated at $635^{\circ} \mathrm{C}$ and tested at $400^{\circ} \mathrm{C}$ shows very little effect due to the dose.

These results show that the YS of the unirradiated room temperature test samples and the at $300^{\circ} \mathrm{C}$ tested material is not significantly different $(30 \mathrm{MPa})$ but the temperature difference of $100^{\circ} \mathrm{C}$ on the unirradiated sample led to $\sim 60 \mathrm{MPa}$ difference in YS (510 MPa YS for the test performed at $400^{\circ} \mathrm{C}$ and $570 \mathrm{MPa}$ for the test performed at $300^{\circ} \mathrm{C}$ ). It can be seen that a change in testing temperature has a strong influence on the value gained in the tensile test above $300^{\circ} \mathrm{C}$. It is obvious in analyzing the tensile test results of the irradiated material that the most irradiation

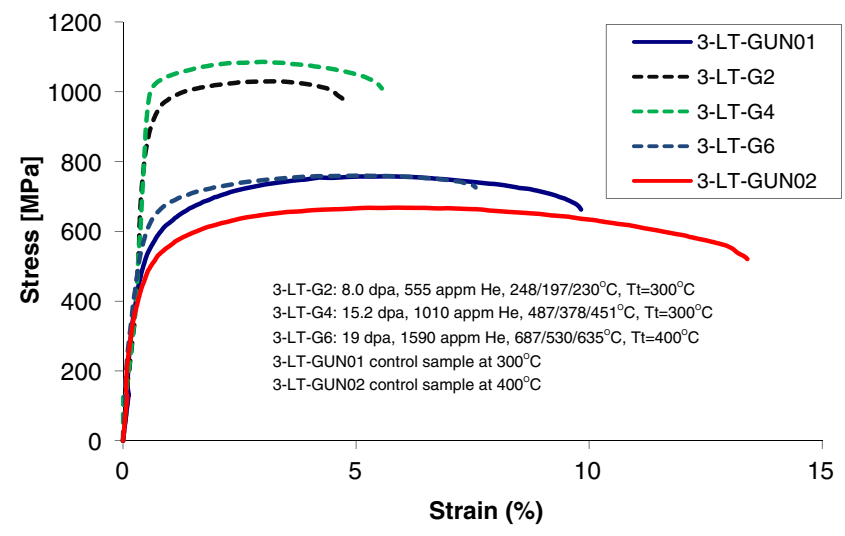

Fig. 4 Tensile test results on HT-9 irradiated in STIP 
and He induced hardening occurs at the low dose and low temperature sample. An increase of the dose by a factor of two and $\sim 220^{\circ} \mathrm{C}$ higher average irradiation temperature dose not lead to a significantly higher radiation induced hardening. Apparently between $450^{\circ} \mathrm{C}$ and $635^{\circ} \mathrm{C}$ average irradiation temperature is where the induced irradiation damage begins to annihilate. The He will not diffuse out of the bulk sample at $635^{\circ} \mathrm{C}$ and only can form larger bubbles and migrate to grain boundaries. But it can be said that most of the radiation damage does heal (temperature is close to annealing temperature) out leading to the conclusion that the He does not have an overwhelming strong effect in the radiation induced hardening than the radiation damage itself.

Micro Mechanical Tests on HT-9 Irradiated at STIP and Comparison to the Tensile Testing

Figure 5 combines data from the micromechanical tests (micro hardness and micro pillar) on the same graph as well as the tensile test results. It can be seen that the micro mechanical test results follow the same trend as the test results obtained through macroscopic tensile testing. It has to be noted that the test temperature of the tensile tests were performed at $300^{\circ} \mathrm{C}$ and $400^{\circ} \mathrm{C}$ while the test results on the micro mechanical tests were performed at room temperature. This explains the lower values observed in the tensile test results. The fact that the drop in YS at the $19 \mathrm{dpa}$ sample using micro mechanical testing is not as strong as the drop in YS shown by tensile testing is explained by the fact that the 19 dpa tensile test was performed at $400^{\circ} \mathrm{C}$ and not at $300^{\circ} \mathrm{C}$. Therefore, the reduction in yield stress observed in the tensile tests is affected by the higher temperature used for the tensile tests as well as the increased irradiation temperature.

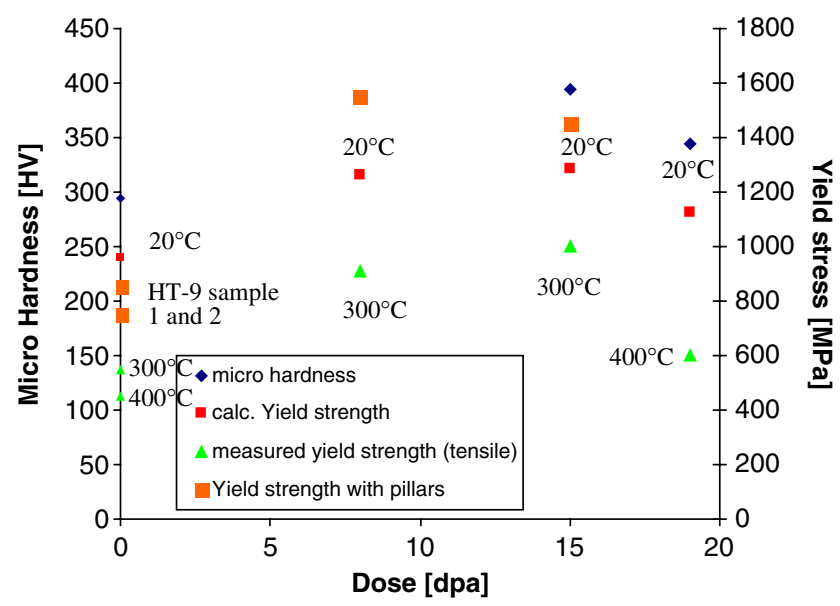

Fig. 5 Summary of the micro hardness, micro compression and tensile tests performed on the STIP samples. The micro compression testing was not performed at the 19 dpa sample
It has also to be considered that the micromechanical testing samples only test a very small sample volume and therefore errors from texture effects can occur especially on the micro compression tests. It is assumed that the FIB damage is negligible since the Ga beam only penetrates $20 \mathrm{~nm}$ or less while the sample cross section is $8 \mu \mathrm{m} \times 8 \mu \mathrm{m}$.

Interestingly, it can be seen that above $10 \mathrm{dpa}$ the YS increase is not increasing as strongly as at lower dose. All three testing techniques show this trend. Again, in addition to the dose the irradiation temperature has to be considered. The fact that the $15 \mathrm{dpa}$ samples have seen an average irradiation temperature of $451^{\circ} \mathrm{C}$ is significantly higher than the average irradiation temperature of $230^{\circ} \mathrm{C}$ for the $8 \mathrm{dpa}$ sample. However, $451^{\circ} \mathrm{C}$ is not high enough to fully anneal the damage produced from radiation. Therefore the weak increase in YS at $15 \mathrm{dpa}$ is attributed to both a slight annealing of the radiation damage due to higher irradiation temperature as well as reaching the dose plateau from a saturation in defect density. Although it is difficult to separately address the hardening caused from helium buildup, this 15 dpa sample may also be showing some hardening from the increased helium content.

\section{Local Electrode Atom Probe Measurements}

Several LEAP samples were manufactured from the $8 \mathrm{dpa}$ sample. All samples were cut from single grains Fig. 6 (a)

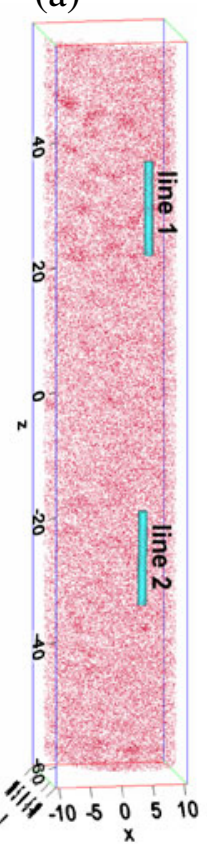

(b)

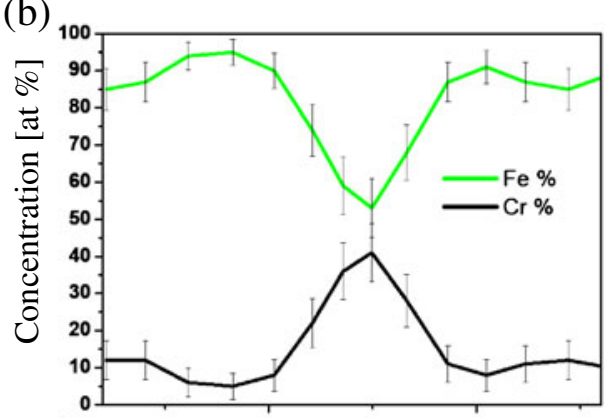

(c) 1

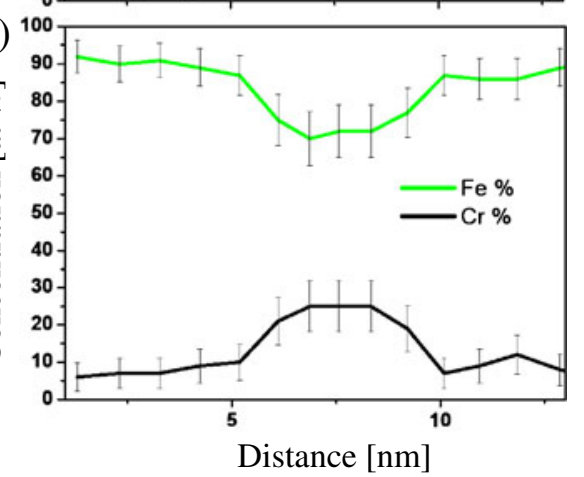

Fig. 6 Local Electrode atom probe results gained on the 8 dpa STIP sample. Significant local $\mathrm{Cr}$ enrichments were found in different locations (marked in atom probe map) 
depicts a subvolume of an atom probe measurement (only $\mathrm{Cr}$ atoms are shown). It is obvious that $\mathrm{Cr}$ is not randomly distributed. Local enrichments of $\mathrm{Cr}$ are visible as can be seen in Fig. 6(a). Figure 6(b) and c illustrate two different line profiles indicated in Fig. 6(a). As can be seen from these line profiles, $\mathrm{Cr}$ enrichments containing $40-50 \mathrm{at} \% \mathrm{Cr}$ can be found Fig. 6(b). Line profiles were used to estimate the chemical composition and subsequent local enrichments. Similarly, areas with lower $\mathrm{Cr}$ concentrations could be found [see Fig. 6(c)].

It is known from the literature that a fully aged alpha-prime $\left(\alpha^{\prime}\right)$ phase contains $\sim 95 \% \mathrm{Cr}$. Here only $50 \% \mathrm{Cr}$ were found. However, most data on $\alpha^{\prime}$ at these temperatures found in the phase diagrams are based on modeling. Very little quantitative experimental data is available especially on low temperature irradiated materials. Considering the low temperature of these irradiations $\left(230^{\circ} \mathrm{C}\right.$ on average $)$ it is possible that because of the slow kinetics the formation of the fully stoichiometric $\alpha^{\prime}$ phase is not finished after 18 months under this condition. Nevertheless, more thorough atom probe investigations of irradiated samples are needed to investigate the true composition of the $\mathrm{Cr}$ enrichments.

\section{Summary}

This work compares three different techniques of mechanical testing on materials irradiated in STIP. It is found that all three techniques can be used to measure the changes in mechanical properties due to radiation. Using the small scale mechanical testing, a much smaller volume of radioactive material can be tested allowing for more efficient and safer handling of the samples. In addition this work shows clearly that a much wider variety of tests can be performed on the same sample allowing one to access more data with a very small volume of material. Work is currently ongoing in development of similar techniques to perform the same small scale mechanical tests at different temperatures. This will allow significant insight into the thermal energy required to anneal radiation damage out of material as a function of the numerous material and system parameters (chemistry, microstructure, dose rate, etc).

In addition in this work it has been clearly shown that any LEAP sample which can be cut by FIB is well below any detectable limit and LEAP measurements can be performed outside controlled areas without major radiological precautions. The LEAP measurements performed here show that after $8 \mathrm{dpa}$ irradiation at $230^{\circ} \mathrm{C}$ small $\mathrm{Cr}$ rich clusters were found.

Acknowledgment This work was performed, in part, at the Center for Integrated Nanotechnologies, a US Department of Energy, Office of Basic Energy Sciences user facility. Los Alamos National Laboratory, an affirmative action equal opportunity employer, is operated by Los Alamos National Security, LLC, for the National Nuclear Security Administration of the US Department of Energy under Contract DE-AC52-06NA25396. The University of Leoben, and in particular Dr. Harald Leitner and Prof. Dr. Helmut Clemens were supportive of this work by allowing an extensive student exchange. The Energy Frontier Research Center (EFRC) lead by Michael Nastasi allowed for further information exchange and support.

Open Access This article is distributed under the terms of the Creative Commons Attribution Noncommercial License which permits any noncommercial use, distribution, and reproduction in any medium, provided the original author(s) and source are credited.

\section{References}

1. Dai Y, Bauer GS (2001) J Nucl Mater 296:43-53

2. Klueh L, Nelson AT (2007) J Nucl Mater 371:37-52

3. Maloy SA, Romero T, James MR, Dai Y (2006) J Nucl Mater 356:56-61

4. Lillard RS, Paciotti M, Tcharnotskaia V (2004) J Nucl Mater 335:487

5. Gelles DS (1990) Metall Trans A 21:1066

6. Hosemann P, Swadener JG, Welch J, Li N (2008) J Nucl Mater 377:201-205

7. Hosemann P, Hawley ME, Koury D, Welch J, Johnson AL, Mori G, Li N, Maloy SA (2008) J Nucl Mater 381:211-215

8. Hosemann P, Kabra S, Stergar E, Maloy SA. accepted at J Nucl Mater, doi:10.1016/j.jnucmat.2010.05.005

9. Dai Y, Jia X, Thermer R, Hamaguchi D, Geissmann K, Lehmann E, Linder HP, James M, Gröschel F, Wagner W, Bauer GS (2005) J Nucl Mater 343:33-44

10. Tong Z, Dai Y (2009) J Nucl Mater 385:258-261

11. Jung P, Hishinuma A, Lucas GE, Ullmaier H (1996) J Nucl Mater 232:186

12. Hosemann P, Swadener JG, Kiener D, Was GS, Maloy SA, Li N (2008) J Nucl Mater 375:135-143

13. Hosemann P, Lei P, Stergar E, Dai Y, Pouchon M, Maloy SA. Accepted to J Nucl Mater. ICFRM-14 conference proceedings

14. Kelly TF, Thompson K, Marquis EA, Larson DJ (2006) Micorscopy today $34-40$

15. Thompson K, Larson DJ, Ulfig RM (2005) Micro Microanal 11:882

16. Rachbauer R, Massl S, Stergar E, Felfer P, Mayrhofer PH (2010) Surf Coat Technol 204:1811-1816

17. Uchic MD, Dimiduk DM, Florando JN, Nix WD (2004) Science 305:986-989

18. Kiener D, Motz C, Schöberl T, Jenko M, Dehm G (2006) Adv Eng Mater 8:1119-1125

19. IVAS software, IMAGO INC 